\title{
Primary epiploic appendagitis: Laparoscopic diagnosis and treatment
}

\author{
Rachel M. Gomes • Sivalingam Perumal • \\ Saravana S. Kumar • Palanisamy Senthilnathan • \\ Ramakrishnan Parthasarathi - Subbiah Rajapandian • \\ Chinnusamy Palanivelu • Palanivelu Praveen Raj
}

Published online: 19 February 2015

(C) Indian Society of Gastroenterology 2015

Sir,

The diagnosis of primary epiploic appendagitis was rarely made preoperatively, but nowadays, with improved imaging, this is possible in many cases. We read with interest the article "Primary epiploic appendagitis: Reconciling CT and clinical challenges" published in your Journal [1]. The authors describe 15 cases of primary epiploic appendagitis which were diagnosed on CT scan, treated conservatively and advised for follow up. Only 10 patients completed the advised follow up schedule of a CT scan at 2 weeks, 3 months and 6 months to look for resolution. Complete resolution was noted in three patients at 2 weeks, in six patients at 3 months and in one patient at 6 months.

We at our institution treat epiploic appendagitis surgically by the laparoscopic method. The optimum treatment for this condition is still a matter of controversy. Most authors state that if diagnosed preoperatively, it should be managed conservatively with antibiotics and analgesics. We wish to point out several issues in clinical practice with this approach. This condition is relatively rare with a possibility of misdiagnosis especially if there is significant colonic wall thickening on imaging in spite of its characteristic imaging features. Symptoms resolve slowly in 7-10 days with imaging resolution taking even up to 6 months as was also noted in this study. The latter adds to diagnostic confusion and makes it necessary that the patient be subjected to the anxiety and expense of several follow up imagings lest a more alarming diagnosis is missed out. Many patients are lost to follow up. On the other hand, laparoscopic diagnosis and treatment offers an excellent option. It confirms the diagnosis without the morbidity of open surgery and allows for surgical removal with subsequent rapid recovery. Histopathological confirmation is made, and there is no need for follow up. Laparoscopy should be strongly considered as a primary treatment option for primary epiploic appendagitis.

\section{Reference}

1. Saad J, Mustafa HA, Elsani AM, Alharbi F, Alghamdi S. Primary epiploic appendagitis: Reconciling CT and clinical challenges. Indian J Gastroenterol. 2014;33:420-6.

R. M. Gomes · S. Perumal · S. S. Kumar · P. Senthilnathan •

R. Parthasarathi $\cdot$ S. Rajapandian $\cdot$ C. Palanivelu $\cdot$

P. Praveen Raj $(\square)$

Department of Minimal Access Surgery, GEM Hospital and

Research Centre, Coimbatore 641 045, India

e-mail: praveenraj@me.com 